\title{
Measurement of Particles in Aerosols - Challenge and Solutions
}

\author{
Jürg Schlatter
}

\begin{abstract}
Ambient air is a mixture of gases, droplets and solid particles and therefore a typical aerosol. Natural and anthropogenic processes govern the actual composition of the air. In some cases the generated components are toxic and should be avoided. The key issue of the identification, reduction, and limitation of a component is the measurement. Unfortunately an aerosol cannot be stored in containers and is not available as a reference material. Therefore the calibration of measuring instruments is fundamental to obtain reliable results. Because various measuring principles are used for many applications such as cleanrooms, engine emissions, ambient air etc., the calibration procedures must be adopted. Metrological institutes such as METAS have recognised the need for reliable reference values and have initiated calibration services and international collaboration. Therefore international harmonised reference values for particle number concentration and particle size are available.
\end{abstract}

Keywords: Aerosol · Airborne particles · Number concentration · Particle mobility

\section{Introduction}

Ambient air is a mixture of gases, droplets and solid particles. Natural and anthropogenic processes govern the actual composition of the air. In some cases the generated components are toxic and should be avoided. The key issue of the identification, reduction, and limitation of a component is the measurement. Four examples show this:

Large cities suffer from smog and heavy air pollution. ${ }^{[1,2]}$ Less traffic and optimised combustion processes for heating and thus lower emissions of ultrafine particles are demanded. Who knows how to measure the correct particle concentration? Ambient measurements are necessary to examine the impact of administrative measures. Calibrated measuring instruments deliver the basic information on which decisions are made.

Future diesel vehicles and engines are equipped with certified particulate traps. Who can guarantee and certify the traps' efficiency? Requirements for particle traps define retention coefficients for nanoparticles. ${ }^{[3]}$ Calibrated instruments are needed to give reliable values for the certification.

Nanostructures have many applications and reveal many new opportunities. ${ }^{[4]}$ Research and production activities need particle-free ambient conditions. Who quantifies the quality of the production? The smaller the structure is, the cleaner the environment has to be. Calibrated particle counters indicate the quality status of cleanroom environments. Therefore particle measurements may guarantee safe operations in such protected areas.

Society demands a high standard of public safety. Danger from germs, viruses, bacteria and fallout can be recognised by the measurement of aerosols in critical zones such as food technology, medical areas and public places, e.g. airports, train stations, customs. Exposure hazards are detected with particle detectors. Reliable measuring results enable adequate security measures to be taken.
Measurement instruments and their calibration procedures are available for the above challenges. Because the measurement tasks have a wide range of application, the measurand, the sampling, the instrument, and also the calibration procedure of the instrument must be chosen carefully in order to obtain reliable results on which decisions can be based in the most efficient way.

\section{Measurands for Particles}

An aerosol is a mixture of liquid and solid airborne particles in a gas. The lower size limit of particles in aerosols cannot be clearly defined because nanoparticles can also be large molecules (fullerenes, macromolecules). The size and the number concentration of particles have a wide range (Table).

Since particles may behave similarly to large molecules the particle density is also a function of temperature and pres-

Table. Examples of number concentrations and particle sizes

Typical number concentration
$\begin{aligned} & \text { Diesel exhaust } \\ & \text { Urban air }\end{aligned}$
Rural air
$\begin{aligned} & \text { Jungfraujoch } \\ & 10000000000 \mathrm{~cm}^{-3}\end{aligned}$
$\begin{aligned} & \text { Clean room class } 9 \\ & (>0.5 \mu \mathrm{m})\end{aligned}$
$\begin{aligned} & \text { Clean room class } 6 \\ & (>0.1 \mu \mathrm{m})\end{aligned}$

Typical size range

Water molecule

$0.3 \mathrm{~nm}$

Viruses

$1 \mathrm{~nm}-5 \mathrm{~nm}$

Tobacco and

Engine smoke

$10 n m-500 n m$

Bacteria

$0.5 \mu \mathrm{m}-50 \mu \mathrm{m}$

Federal Office of Metrology METAS

Section Analytical Chemistry

Particle Laboratory

Lindenweg 50

$\mathrm{CH}-3003$ Bern-Wabern

Tel.: +4131323 3382

Fax: + 41313233210

E-mail: juerg.schlatter@metas.ch
(>0.1 $\mu \mathrm{m})$

Coal dust

$1 \mu \mathrm{m}-100 \mu \mathrm{m}$

Pollen particle 
sure. Therefore reference conditions for temperature and pressure are essential for each indication for the particle number concentration.

The amount of particles can be indicated as a quantum in a unit volume gas. For the quantum various measures are commonly used. These are the number of particles, the mass of particles, their surface area, even a specific fraction of surface area associated with chemical reactivity is possible. METAS focuses on the particle number concentration measurement, but future work is also planned with particle mass.

Solid particles usually are not spherical and have various shapes. Therefore the size of particles cannot be indicated by a geometrical diameter. To visualise a particle as a sphere is a rough simplification. Nevertheless an equivalent diameter is widely used to characterise particle size. ${ }^{[5]}$ Liquid aerosol particles are nearly always spherical, but also for these particles equivalent diameters are needed to define their size.

The equivalent diameter is defined as the diameter of a spherical particle with the same specified property as the considered particle. The measuring method defines the considered property of the particle. Various equivalent diameters of a particle due to various properties are normally not equal and cannot be transformed from one to another (see examples in Fig. 1).

Particle analysers are often used for sizing the mobility diameter, the aerodynamic diameter, and the optical diameter. The mobility of the particle is a measure for the diffusive dispersion and deposition. Diffusion and therefore mobility is the dominant parameter for the deposition of nanoparticles in the respiratory system. The aerodynamic diameter of a particle is a measure for particle deposition by impaction. It defines for instance the deposition rate of fine and coarse particles in the respiratory system. As the name implies, the optical diameter gives a measure of the optical behaviour of the particles as light absorption or light scattering. The optical properties are not directly linked to health effects except for safety reasons, if the optical density affects visibility in road traffic.

\section{Particle Size Distribution Parameter}

The size of airborne particles is not uniform; natural particles are not monodisperse. The sizes of a polydisperse aerosol range over two or more orders of magnitude. Particle sizing instruments can classify the particles according to equivalent diameters and give the measurement results in a particle size distribution. The size

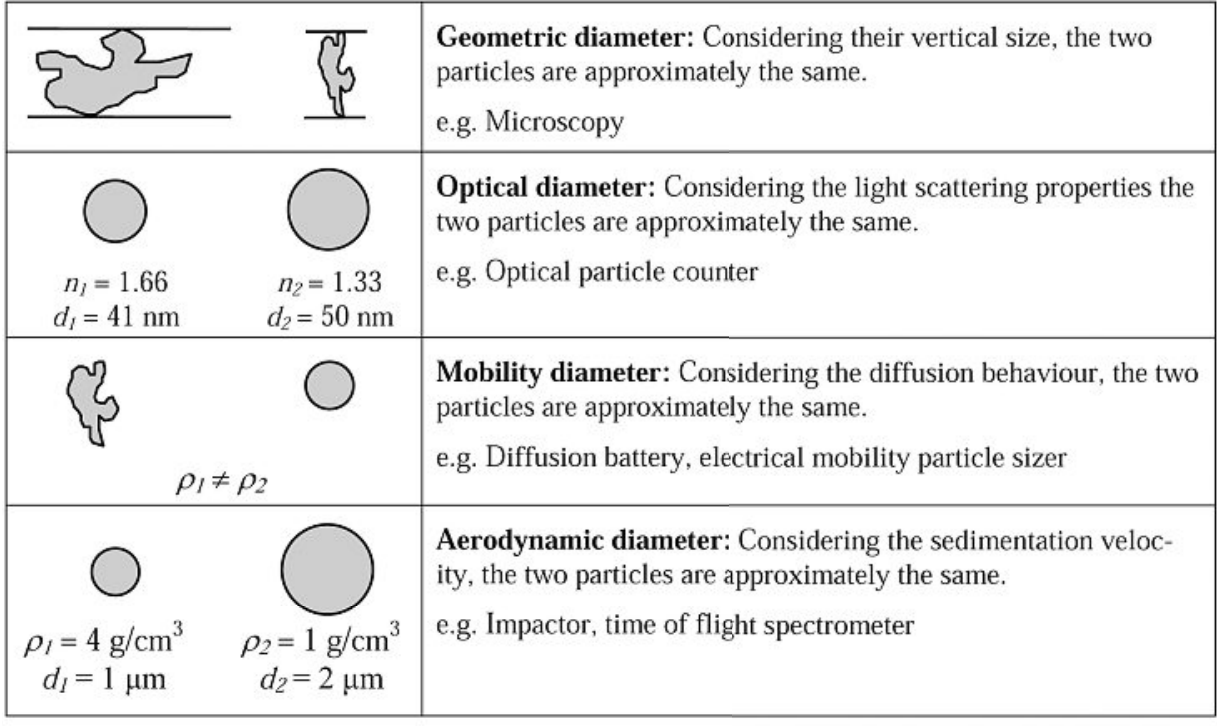

Fig. 1. Comparison of different measurands for particle sizing.

distribution offered by the instruments' software is given as a table of values. Particle measurements are normally given with logarithmic spaced size bins at geometric mean diameters $d_{1}, d_{2}, d_{3} \ldots d_{n}$ with $d_{i+1} / d_{i}$ $=$ constant

For the particle number concentration for each bin $i$ two measures are used: First is the average differential number concentration density $c_{i}$ and second is the number concentration $C_{i}$ in the size bin (also indicated as $\mathrm{d} W$ ). Both can be derived from the cumulative distribution curve $Q(d)$ (often indicated as $N(d)$ ) that indicates the number concentration for particles having diameters less than $d$.

Differential number concentration density at the diameter $d_{i}$ is given in Eqn. (1).

$$
c_{i}^{(\log )}=\frac{\mathrm{d} Q\left(d_{i}\right)}{\mathrm{d} \log (d)} c_{i}^{(\mathrm{ln})}=\frac{\mathrm{d} Q\left(d_{i}\right)}{\mathrm{d} \ln (d)}
$$

Here the choice of the basis for the logarithm is essential, but the concentration density can be transformed as $c_{i}^{(\log )}=c_{i}^{(\mathrm{ln})} \cdot \ln (10)$.

The bin particle number concentration is given in Eqn. (2).

$$
C_{i}=\frac{c_{i}^{(\log )}}{2} \log \left(\frac{d_{i+1}}{d_{i-1}}\right)=\frac{c_{i}^{(\ln )}}{2} \ln \left(\frac{d_{i+1}}{d_{i-1}}\right)
$$

The table of values $\left(d_{i}, c_{i}^{(\log )}\right)$ or $\left(d_{i}, c_{i}^{(\mathrm{ln})}\right)$ or $\left(d_{i}, C_{i}\right)$ is used to calculate size distribution coefficients such as total number concentration, mode, geometric mean, median, and geometric standard deviation. Commonly the number concentration is indicated as concentration density to the basis 10 of the logarithm $(\mathrm{d} Q / \mathrm{d} \log (d))$.

The total number concentration is the number of particles in the volume unit $\left(\mathrm{cm}^{-3}\right)$ within the measured size range (Eqn. (3)).

$$
C=\sum_{i=1}^{n} \frac{c_{i}^{(\log )}}{2} \log \left(\frac{d_{i+1}}{d_{i-1}}\right)=\sum_{i=1}^{n} \frac{c_{i}^{(\mathrm{ln})}}{2} \ln \left(\frac{d_{i+1}}{d_{i-1}}\right)=\sum_{i=1}^{n} C_{i}
$$

The mode diameter is the diameter associated with the highest point on the size distribution curve. The mode $\hat{\mathrm{d}}^{(\mathrm{ln})}$ for a size distribution with linear diameter scale is much smaller than the mode $\hat{\mathrm{d}}^{(\mathrm{n})}$ for a size distribution with a logarithmic diameter scale. Here we use the mode in the logarithmic scale (Eqn. (4)).

$$
\hat{d}=\hat{d}^{(\ln )}=\hat{d}^{(\log )}=d_{m} \text { with } m: c_{m}=\max \left(c_{i}\right)
$$

The geometric mean is defined as the $n$th root of the product of $n$ values. For particle distributions the geometric mean diameter is equivalent to the anti-log of the concentration-weighted mean of the natural logarithmic diameters (Eqn. (5)).

$$
d_{g}=\exp \left(\frac{1}{C} \sum_{i=1}^{n} C_{i} \ln \left(d_{i}\right)\right)
$$

The count mean diameter is defined as the arithmetic mean of the particle diameter in the system (Eqn. (6)).

$$
\bar{d}=\frac{1}{C} \sum_{i=1}^{n} C_{i} d_{i}
$$

The count median diameter is defined as the diameter for which one-half the total number of particles are smaller and onehalf are larger (Eqn. (7)). 


$$
\begin{aligned}
d_{50}=d_{m} \text { with } m: & \sum_{i=1}^{m-1} \frac{c_{i}}{2} \log \left(\frac{d_{i+1}}{d_{i-1}}\right) \\
& \approx \sum_{i=m+1}^{n} \frac{c_{i}}{2} \log \left(\frac{d_{i+1}}{d_{i-1}}\right)
\end{aligned}
$$

A better estimation for $d_{50}$ can be obtained with the assumption of a constant $c_{i}$ within size bin $m$ and interpolation of the diameter within the boundaries of the size bin.

The geometric standard deviation GSD or $\sigma_{g}$ is the standard deviation of the distribution with a natural logarithmic diameter scale (Eqn. (8)).

$$
\sigma_{g}=\exp \left(\sqrt{\frac{1}{C} \sum_{i=1}^{n} C_{i}\left(\ln \left(d_{i}\right)-\ln \left(d_{g}\right)\right)^{2}}\right)
$$

\section{Particle Modification}

An aerosol cannot be stored in containers, because the particle number concentration and the particle size distribution do not remain constant. The underlying processes for this modification are known as aerosol aging. They have various driving forces and various time constants. The main processes are evaporation/condensation, diffusion, impaction, sedimentation, coagulation, thermophoresis, and electrical attraction. The time coefficients of these processes depend on the particle size. Thus smaller particles have higher diffusion losses or electrostatic losses, and larger particles are dominating the impaction and sedimentation. Fig. 2 summarises the main processes for particle modification.

\section{Measuring Methods}

\subsection{Particle Counter}

The particle number concentration can be measured directly with a particle counter or calculated as the integration of the particle size distribution that is measured with a particle size analyzer.

Condensation particle counters (CPC) are often used for particles between several $\mathrm{nm}$ and $1 \mu \mathrm{m}$. The measuring principle of a CPC is based on (a) the condensation and growth of a working liquid (alcohol or water) around each particle in a supersaturated environment and (b) the detection of the generated droplets with light scattering. Particle concentrations below $10000 \mathrm{~cm}^{-3}$ can be detected by counting the single scattered light flashes from particles passing through a continuous laser beam. At higher concentration the probability of

\begin{tabular}{|c|c|}
\hline $\begin{array}{l}\text { Volatilization and condensation: Aerosol particles (black dots) } \\
\text { often consist in condensate volatile compounds that are in equilib- } \\
\text { rium with the gas phases (e.g. VOC, volatile inorganic salts as } \\
\text { ammonium salt). Changes of temperature, pressure or a dilution } \\
\text { with fresh air may change the partitioning on the particles and the } \\
\text { gas phases (upper graph: volatilisation, lower graph: condensa- } \\
\text { tion). Thus the chemical structure and the size of particles change. }\end{array}$ & \\
\hline $\begin{array}{l}\text { Diffusion: Aerosol particles (dots) leave the streamline (black } \\
\text { lines) of the gas because of their Brownian motion (curve) and } \\
\text { touch the wall of the container or of an obstacle (circle). There } \\
\text { they stick. }\end{array}$ & \\
\hline $\begin{array}{l}\text { Impaction: Aerosol particles (dots) in principle follow the } \\
\text { streamlines of the gas (black lines). When the density of particles } \\
\text { is high and the streamline curves narrowly, the particles keep the } \\
\text { direction due to their inertia and collide with an obstacle. }\end{array}$ & \\
\hline $\begin{array}{l}\text { Sedimentation: Larger aerosol particles (typically over } 1 \mu \mathrm{m} \text { ) are } \\
\text { prone to the sedimentation process. Particles with higher density } \\
\text { (black dots) follow gravity faster than lighter particles with the } \\
\text { same diameter (grey dots). Especially in horizontal streamlines } \\
\text { particles leave the gas flow. }\end{array}$ & $\bar{\equiv}$ \\
\hline $\begin{array}{l}\text { Coagulation: Aerosols with very high particle number concentra- } \\
\text { tion are not stable. The chance of a particle to collide is propor- } \\
\text { tional to the number concentration. Each collision ends in sticking } \\
\text { together, reducing the number concentration, and increasing the } \\
\text { particle size. }\end{array}$ & \\
\hline $\begin{array}{l}\text { Electrostatic Force: Electrically charged aerosol particles ex- } \\
\text { perience electrostatic forces in an electrical field. This field can be } \\
\text { caused by surface charges on non conductive materials. Because a } \\
\text { neutral aerosol always contains particles with both electrical po- } \\
\text { larities, potential inhomogeneities of the surface may cause effi- } \\
\text { cient particle loss. }\end{array}$ & \\
\hline $\begin{array}{l}\text { Thermophoresis: Aerosol particles in a temperature gradient } \\
\text { (flame and ice crystal) move by diffusion preferably to the colder } \\
\text { environment. The higher the mobility is (grey dots compared to } \\
\text { black dots) the faster the particle moves. }\end{array}$ & 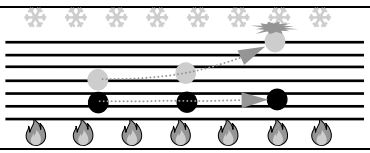 \\
\hline
\end{tabular}
multiple particles in the measuring zone (coincidence) and the measurement un-

Fig. 2. Overview of particle transformation processes.

certainty increase. In that case the number concentration is taken from the scattered light intensity via a calibration curve. This measurement principle assumes the condensation on each single particle with a probability close to a hundred percent. This assumption cannot be fulfilled for particles with sizes at the lower detection limit and the detection efficiency drops to zero for small particles. The measurement and the certification of this efficiency as a function of particle size is an essential task for reliable measurements.

A Faraday cup electrometer (FCE) can expand the size range for particle measurement to smaller sizes, but requires both known electrical charges on each particle and high number concentrations (above $1000 \mathrm{~cm}^{-3}$ ). In a FCE the particles are retained in a filter that is encapsulated in a Faraday cup. The electrical charge from the particles is measured as a current in an electrometer and together with the charge information and the aerosol flow the particle number concentration can be calculated. The uncertainty of measurements with FCE is dominated by the uncertainty of the number of charges per particle and the drift and noise of the electrometer.

Both CPC and FCE are also used together with pre-separation according the particle size in a differential mobility par- ticle sizer or a diffusion battery that fractionises the particles based on different diffusivities.

\subsection{Particle Sizer}

The measurement of the particle size distribution combines knowledge of the number concentration and size information. Particle size distributions are often measured with an optical shape analyser, an optical particle counter, an electrical mobility particle sizer, a time of flight spectrometer, a diffusion battery, or an electrical cascade impactor. The foremost challenge of particle sizing is the choice of the appropriate size measure. Each measuring method has a different measurand that is not equivalent to the others (Fig. 1).

Industrial applications mostly use only a few of the above measuring techniques. Optical particle counters are required for cleanroom surveys and continuous exhaust analysis. Impactor and electrical mobility analysers can be found in the automotive industry, for health surveys, and for combustion research. Due to this need METAS focuses on the optical and the mobility diameter. Here only optical particle counters and electrical mobility particle sizers shall be discussed.

In an optical particle counter (OPC) the particles are irradiated by monochro- 
matic or polychromatic light in a measuring zone. ${ }^{[6]}$ Each single particle scatters the light, which is measured under one or several fixed scattering angles. The intensity of the light gives information of the optical particle diameter and the rate of light flashes together with the flow rate defines the number concentration. Because the optical diameter is a combination of geometric diameter, shape and optical property (refractive index) of the particles, the size measurement must be adjusted with standard particles. Polystyrene spheres with a certified diameter are established as size standards. Nevertheless the harmonised size adjustment does not prevent different constructions of OPC (optical geometry, light colour) from bias, if they are used with a real aerosol. OPC can measure aerosols with diameters larger than $0.1 \mu \mathrm{m}$. Finally size calibration is only one part of the OPC verification. In many cases the number concentration, also called efficiency measurement, is not calibrated and therefore is not traceable to national standards.

The electrical mobility analyser consists of a cylindrical condenser tube. ${ }^{[7]}$ The particles are fed at the outer wall into a laminar flow of clean air. Because of a voltage between the outer wall and the inner wall charged particles are deflected by the electrical field. Small particles having a large electrical mobility move faster to the central electrode than large particles. A fraction with a defined electrical mobility leaves the condenser through a slit in the central electrode and enters the particle counter (CPC or FCE). Slowly changing the voltage in the condenser allows the selected particle mobility to be scanned within the particle size range. The size distribution can be calculated from the particle counter time series. The typical size range of an electrical mobility analyser goes from a $5 \mathrm{~nm}$ to about $1 \mu \mathrm{m}$ and one scan lasts from 1 to 3 minutes. This measuring procedure needs many fitting parameters that are defined by the construction. Traceable results can only be obtained if the instrument has been calibrated to certified standards with spherical particles and certified particle counters.

\section{Calibration of Particle Analyzers at METAS}

\subsection{Cleanroom Particle Counters}

Cleanrooms control airborne particulate contamination to levels appropriate for accomplishing contamination-sensitive activities. The international standard ISO 14644-1 assigns classification levels to be used for the specification of air cleanliness in cleanrooms. ${ }^{[8]}$ It prescribes the standard method of testing as well as the procedure for determining the concentration of air-

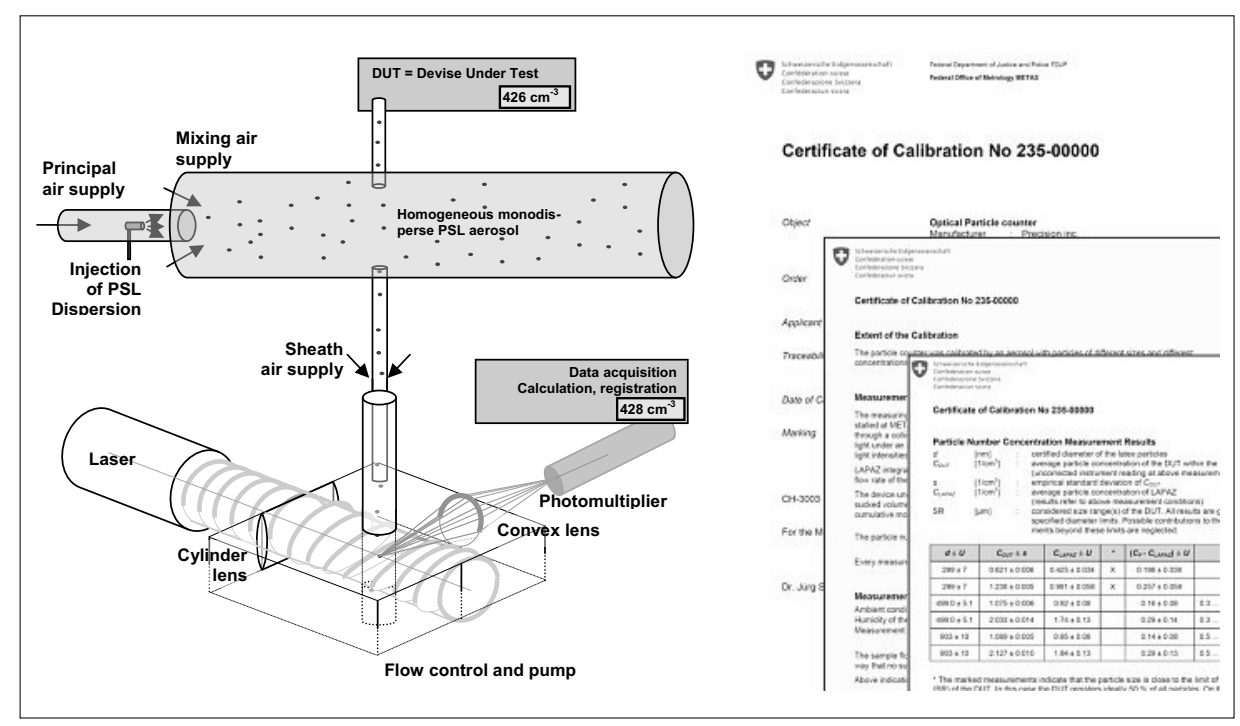

Fig. 3. Scheme of aerosol generator, homogenisator, METAS reference optical particle counter LAPAZ (Laser PArtikel Zähler) and device under test; example of METAS calibration certificate.

borne particles. The particle measuring instruments fulfil the requirements for the state of the technology and are calibrated annually. ${ }^{[9]}$

For the calibration of optical particle counters METAS operates a calibration bed with an aerosol generator, a homogeniser and a LaserPArtikelZähler (LAPAZ laser particle counter). LAPAZ is a primary standard for measuring particle number concentration in aerosols. ${ }^{[10]}$ It consists of a laser, a measuring cell, a photomultiplier, and the data collection system. Each particle from a free jet of aerosol intersects with the solid-state laser beam and produces a scattered light pulse. The pulses are counted and their amplitudes indicate the particle sizes. For calibration the generator is fed with spherical polystyrene particles. They have diameters between $150 \mathrm{~nm}$ and $5000 \mathrm{~nm}$ and concentrations of below $10 \mathrm{~cm}^{-3}$, with a probe capacity of $50 \mathrm{~L} \cdot \mathrm{min}^{-1}$. Periodically METAS invites participants to calibrate cleanroom particle counters at $0.3 \mu \mathrm{m}, 0.5 \mu \mathrm{m}, 1.0 \mu \mathrm{m}$, and $5 \mu \mathrm{m}$ and number concentration $1 \mathrm{~cm}^{-3}\left(28400 \mathrm{ft}^{-3}\right)$ and $2 \mathrm{~cm}^{-3}\left(56800 \mathrm{ft}^{-3}\right)$ (Fig. 3).

\subsection{Emission Particle Counters}

Particle counters for measurements close to particle sources are designed for high concentrations of small particles. This causes two critical points for particles counters. First, if the particle size is close to the detection limit of the counter, reliable results can only be obtained if the efficiency curve is well known. Therefore new international emission regulation for diesel vehicles ${ }^{[11]}$ defines the efficiency curve of the CPC at particle sizes of $23 \mathrm{~nm}$ and $41 \mathrm{~nm}$ of $50 \%( \pm 12 \%)$ and $>90 \%$ respectively (ref. [11], Appendix 5, clause 1.3.4.9). Secondly at high concentrations coincidence errors influence the measure- ment result. Therefore the cited emission regulation demands an aerosol dilution, in order to keep the concentration below a critical level.

During use at high concentration the CPC collects material on internal surfaces and counting efficiency shifts towards larger particles. Therefore particle counters must be calibrated periodically at various particle sizes at higher concentrations. METAS offers calibration services with combustion particles at diameters between $50 \mathrm{~nm}$ and $200 \mathrm{~nm}$ and concentrations up to $10^{6} \mathrm{~cm}^{-3}$.

\subsection{Ambient Air Particle Analysers}

Particle analysers are used for a wide field of applications: ambient air, exhaust gas from combustion, work places etc. Since the instruments are used at individual settings, the reliability of the results at the settings must be proven by calibration with real aerosols. Since 2002 METAS organises regular calibration workshops for this analysis. ${ }^{[12]}$ The calibration workshop consists of measuring a series of test aerosols using nanoparticles from combustion. The aerosol is generated in the Combustion Aerosol Standard (CAST) by a controlled combustion of propane gas in a diffusion flame. ${ }^{[13,14]}$ The aerosol flow passes dilution steps and a neutraliser $\left({ }^{241} \mathrm{Am}\right.$-source) and is divided to feed the instruments of the participants (Fig. 4).

The measurements with four particle diameters between $50 \mathrm{~nm}$ and $170 \mathrm{~nm}$ are performed in four half-day sessions (Fig. 5). The aerosol is kept constant during a time period of 30 minutes before its concentration is increased. This procedure covers four concentration levels (Fig. 6). For each measurement point the averaged key parameters are evaluated. Every participant operates his instrument on his 


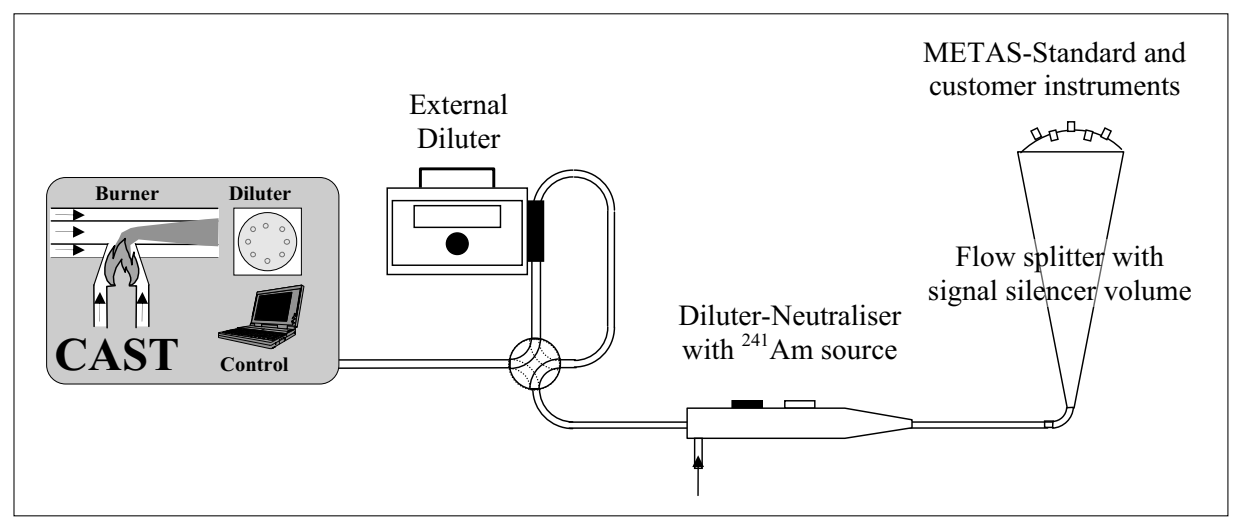

Fig. 4. Scheme of the arrangement and tubing used dilute and split a particle flow generated by a combustion aerosol standard (CAST).
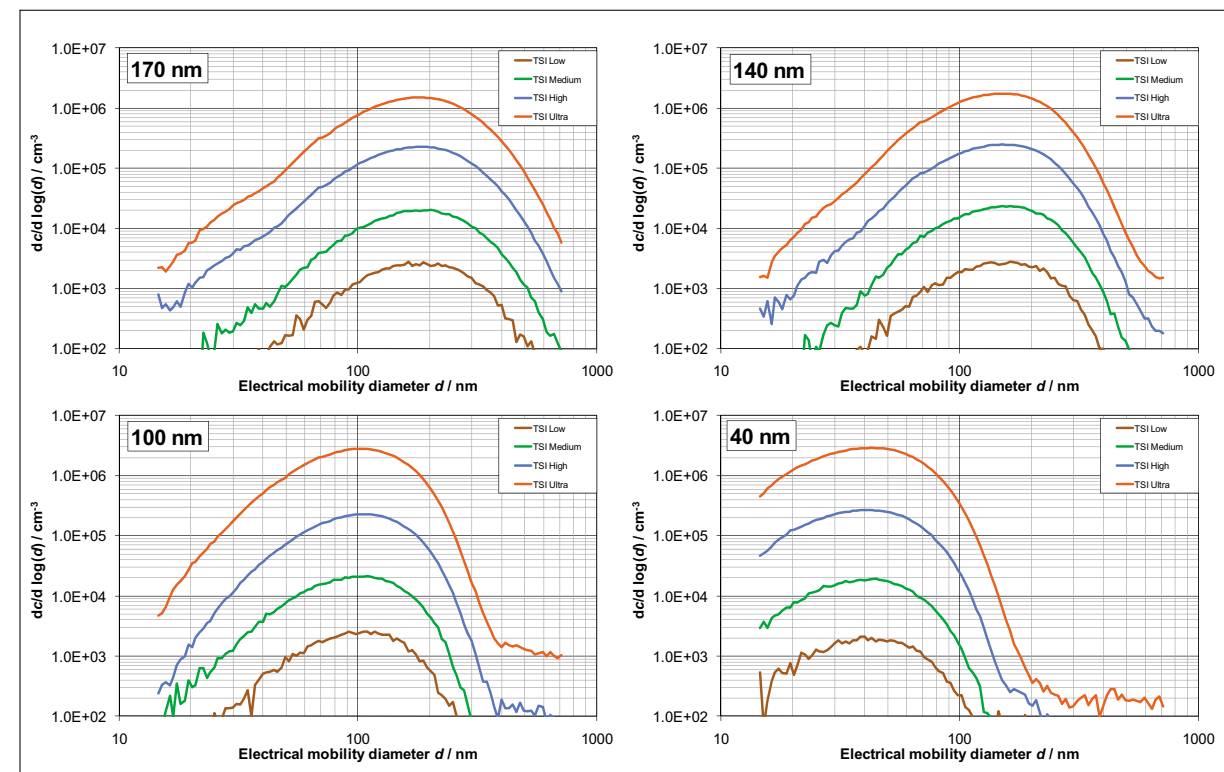

Fig. 5. Averaged number concentration distribution at logarithmic diameter axis for four concentration levels of four diameters of combustion aerosols from CAST.

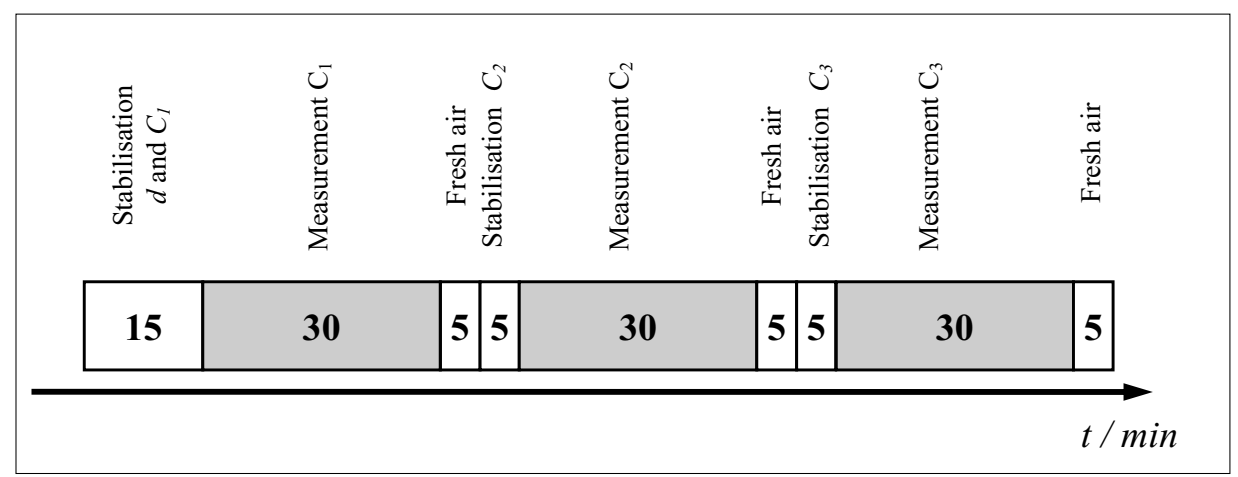

Fig. 6. Procedure scheme for the measurements with one particle size and four concentrations.

own. Therefore the exchange of the results and the experience between the participants permits the individual measuring procedure to be optimised.

In the past the METAS calibration workshop revealed differences between particle measuring principles, instrument types, and instrument setups. Several times hidden instrument failures could be discovered that caused wrong measurement results.

\section{Future Needs of Calibration}

In several areas the need for reliable particle measurements increases. The handling of nanometre-sized particulate structures during production and the knowledge of the goods life cycle require research related to health effects. Concern about air quality requires adequate networks and reliable measuring results. Therefore several metrology institutes all over the world have initiated projects for particle measurement in aerosols. Cooperation for an international size and number concentration standard is established within EURAMET projects 893 (Metrology infrastructure for airborne nanoparticles) and 1027 (Comparison for nanoparticle number concentration and size distribution).

Received: June 26, 2009

[1] Eidgenössische Kommission für Lufthygiene (EKL), 'Feinstaub in der Schweiz. StatusBericht der Eidg. Kommission für Lufthygiene (EKL)', Bern. 141 S, 2007.

[2] C. A. Pope III, M. Ezzati, D. W. Dockery, New Engl. J. Med. 2009, 360, 376.

[3] SNR 277205: 2007, 'Prüfung von Partikelfiltersystemen für Verbrennungsmotoren'.

[4] Bundesamt für Gesundheit BAG, Staatssekretariat für Wirtschaft SECO, Bundesamt für Umwelt BAFU, Aktionsplan 'Synthetische Nanomaterialien', Bericht des Bundesrates vom 9. April, 2008.

[5] W. C. Hinds, 'Aerosol Technology - properties, behaviour, and measurement of airborne particles', John Wiley \& Sons, ISBN 0-471-087262,1982

[6] ISO 21501-4:2007, 'Determination of particle size distribution - Single particle light interaction methods - Part 4: Light scattering airborne particle counter for clean spaces'.

[7] ISO 15900:2009, 'Determination of particle size distribution - Differential electrical mobility analysis for aerosol particles'.

[8] ISO 14644-1:1999, 'Cleanrooms and associated controlled environments - Part 1: Classification of air cleanliness'.

[9] ISO 14644-4:2001, 'Cleanrooms and associated controlled environments - Part 4: Design, construction and start-up'.

[10] J. Schlatter, 'A primary standard for particle number concentrations', in Proceedings of the 12th international metrology congress, 20-23 June, 2005.

[11] ECE/TRANS/WP.29/GRPE/2007/8, Appendix 5, Particle Number Emissions Measurement Equipment.

[12] J. Schlatter, 'Intercomparison of SMPS with CAST particles', in Proceedings of the 7th ETHConference on Nanoparticle Measurement, August, 2003.

[13] L. Jing, 'Standard Combustion Aerosol Generator (SCAG) for Calibration Purposes', in Proceedings of the 3rd ETH Workshop 'Nanoparticle Measurement', ETH Zürich, 9-10 August, 1999.

[14] L. Jing, J. Santos, 'Stability with respect to particle size and concentration of CAST', in Proceedings of the 7th ETH-Conference on Nanoparticle Measurement, August 2003 